\title{
Adaptive Approach to Motion Controller of Linear Induction Motor with Friction Compensation
}

\author{
Chin-I Huang, Student Member, IEEE, and Li-Chen Fu, Fellow, IEEE
}

\begin{abstract}
In this paper, we propose a nonlinear adaptive controller and an adaptive backstepping controller for linear induction motors to achieve position tracking. A nonlinear transformation is proposed to facilitate controller design. In addition, the very unique end effect of the linear induction motor is also considered and is well taken care of in our controller design. We also consider the effect of friction dynamics and employ observer-based compensation to cope with the friction force. A stability analysis based on Lyapunov theory is also performed to guarantee that the controller design here can stabilize the system. Also, the computer simulations and experiments are conducted to demonstrate the performance of our various controller design.
\end{abstract}

Index Terms-Adaptive control, induction motor drives.

\section{NOMENCLATURE}

$a_{1}$

$a_{2}$

$a_{3}$

$a_{4}$

$B$

$\beta$

$c$

$D$

$F_{e}$

$F_{L}$

$\bar{F}_{L}$

$F_{r}$

$i_{q}\left(i_{d}\right)$

$K_{f}$

$L_{m}$

$L_{s}\left(L_{r}\right)$

$M_{m}$

P

$p_{r}$

$\mathrm{p}$

$R_{s}\left(R_{r}\right)$

$v_{r}$

$V_{q s}\left(V_{d s}\right)$

$\lambda_{q}\left(\lambda_{d}\right)$

$\tau$

Manuscript received May 12, 2005; revised December 7, 2006. Recommended by Technical Editor P. R. Pagilla

C. I. Huang is with the Department of Electrical Engineering, National Taiwan University, Taipei 10617, Taiwan, R.O.C. (e-mail: d89921014@ee.ntu.edu.tw).

L. C. Fu is with the Department of Electrical Engineering and Computer Science and Information Engineering, National Taiwan University, Taipei 10617, Taiwan, R.O.C. (e-mail: lichen@ntu.edu.tw).

Digital Object Identifier 10.1109/TMECH.2007.901945

\section{INTRODUCTION}

$\mathbf{N}$ OWADAYS, linear induction motors (LIMs) are widely used in many industrial applications, including transportation, conveyor systems, actuators, material handling, pumping of liquid metal, sliding door closers, etc., with satisfactory performance. The most obvious advantage of linear motors is that they have no gears, and require no mechanical rotary-to-linear converters when translation of payload is concerned. The LIM has many advantages such as simple structure in place of the gear between the motor and the motion devices, reduction of mechanical losses and the size of motion devices, silence, high starting thrust force, and easy maintenance, repairing, and replacement. But for high precision motion performance, the friction problem is one of the significant limitations.

In the early works, Yamamura has first discovered a particular phenomenon of the end effect on LIM [1]. A control method, decoupling the control of thrust and the attractive force of an LIM using a space vector control inverter, was presented in [2], i.e., by selecting voltage vectors of PWM inverters appropriately.

Although the parameters of the simplified equivalent circuit model of an LIM can be measured by conventional methods (noload and locked secondary tests), due to limited length of the machine the realization of the no-load test is almost impossible. Thus, the applicability of conventional methods for calculating the parameters of the equivalent model is limited. In order to measure the parameters, application of the finite element (FE) method for determining the parameters of a two-axis model of a three-phase linear induction motor has been proposed in [3] and [4]. Another method is proposed by removing the secondary [5].

To resolve the unique end-effect problem, speed-dependent scaling factors are introduced to the magnetizing inductance and series resistance in the $d$-axis equivalent circuit of the rotary induction motor (RIM) [6] to correct the deviation caused by the "end effect". On the other hand, there is a thrust correction coefficient introduced by [7] and [8] to calculate the actual thrust to compensate for the end effect. A related method to deal with the problem is that an external force corresponding to the end effect is introduced into the RIM model to provide a more accurate modeling of an LIM under consideration of the end effect as shown in [9]. In another work [10], [21], [22], extra compensating-winding was proposed to alleviate such problem.

Although the end effect is an important issue of the LIM control, there are still many works in the literature that fail to consider it such as [11], [12], and [23]-[25]. In this paper, we will take this as an important issue which cannot be ignored. In addition, as the contact area of bearing in LIM is much larger than that of RIM, the friction term cannot be neglected, inspite 
of the fact that such "friction problem" may not be that critical in high speed applications.

On the other hand, for high precision motion performance, the friction problem leads to one of the significant limitations. Friction varies with temperature, age, and is known to dominate in the low velocity region and during velocity reversal [20]. Failure to compensate for friction in applications may lead to large tracking errors, undesired stick-slip motion, and limit cycles when velocity reversals in the trajectory are required [15], [19]. In order to predict and compensate for the underlying friction, Canudas de Wit has proposed the LuGre model [15], which includes the Stribeck effect, hysteresis, and spring-like characteristics of the stiction, as well as variant break-away force. Furthermore, the adaptive scheme suggested in [16]-[18] has been extended to handle the nonuniform parametric variations of the friction force, and adaptive dynamic friction compensation [20] be applied for trajectory tracking control of robots.

The remainder of this paper is organized as follows. In Section II, we introduce the system model and state the present control objectives. Section III will develop a nonlinear adaptive control of the LIMs presuming that the acceleration signal is measurable. However, in Section IV, we propose an adaptive backstepping approach without measurement of the acceleration signal. To demonstrate the effectiveness of the hereby developed controllers, Section V and VI will, respectively, provide thorough simulations and experiments with satisfactory performance. Finally, Section VII gives some concluding remarks.

\section{PROBLEM Formulation}

To formulate the dynamic model of a linear induction motor, we consider the following assumptions to make the analysis more tractable.

Assumption 1: three phases are balanced.

Assumption 2: the magnetic circuit is unsaturated.

Assumption 3: it is without end effect (we will relax this assumption later in controller design); then the dynamics of the entire system can be rearranged into the following more compact form:

$$
\begin{aligned}
\dot{i}_{q} & =-a_{1} i_{q}+a_{2} \lambda_{q}-\beta p v_{r} \lambda_{d}+c V_{q s} \\
\dot{i}_{d} & =-a_{1} i_{d}+\beta p v_{r} \lambda_{q}+a_{2} \lambda_{d} c V_{d s} \\
\dot{\lambda}_{q} & =a_{3} i_{q}-a_{4} \lambda_{q}+p v_{r} \lambda_{d} \\
\dot{\lambda}_{d} & =a_{3} i_{d}-a_{4} \lambda_{d}+p v_{r} \lambda_{d} \\
M_{m} \dot{v}_{r} & =K_{f}\left(\lambda_{d} i_{q}-\lambda_{q} i_{d}\right)-\bar{F}_{L}-F_{r} .
\end{aligned}
$$

For the meanings of various symbols, readers should refer to the Nomenclature. In this paper, we try to design the speed and position controller for the LIM whose parameters are assumed to be known except the payload. However, some knowledge about the payload structure is available, which is expressed in terms of a second-order differential equation as shown by

$$
\bar{F}_{L}=M_{L}^{\prime} \dot{v}_{r}+b_{L 0}+b_{L 1} v_{r}+b_{L 2} v_{r}^{2} .
$$

Furthermore, the friction force $F_{r}$ in (1) is modeled by the LuGre friction model [20], which uses the following dynamics to express the variation of the friction force variation:

$$
\begin{aligned}
& \frac{d l}{d t}=v_{r}-\frac{\left|v_{r}\right|}{n\left(v_{r}\right)} l \\
& F_{r}=\zeta_{0} l+\zeta_{1} \frac{d l}{d t}+\zeta_{2} v_{r}
\end{aligned}
$$

where $l$ is the friction state that physically stands for the average deflection of the bristles between the two contact surfaces. The friction force parameters $\zeta_{0}, \zeta_{1}$, and $\zeta_{2}$ can be physically explained as the stiffness of bristles, damping coefficient, and viscous coefficient, respectively. In our design, we assume that these three parameters are unknown positive constants. A parameterization of $n\left(v_{r}\right)$ is assumed to be known, and has been proposed to describe the Stribeck effect [20], i.e.,

$$
n\left(v_{r}\right)=F_{c}+\left(F_{s}-F_{c}\right) e^{-\left(\frac{v_{r}}{v_{s}}\right)^{2}}
$$

where $F_{c}, F_{s}$, and $v_{s}$ are the Coulomb friction value, stiction force value, and the Stribeck velocity, respectively.

\section{OBSERVER AND NONLINEAR ADAPTIVE CONTROLLER DESIGN}

In this section, the controller which achieves position tracking of the LIM is proposed. In order to relax the need of flux measurement and to cope with friction, the flux and friction observer will be proposed in Section III-B and C, respectively. The controller should overcome the unknown payload of the LIM while the reasonable assumptions are made. In Section III-D, we propose a position controller to achieve the objective of position tracking with knowledge of the mutual inductance. In Section III-E, an adaptive position controller is proposed to deal with uncertain inductance which achieves the same objective.

\section{A. Analysis of Mechanical Load and End Effect}

The fundamental difference between a rotary induction motor and an LIM is the finite length of the magnetic and electric circuit of the LIM along the direction of the travelling field. The open magnetic circuit causes an initiation of the so-called longitudinal end effects.

In an LIM, as the primary past moves, the secondary past seemingly is continuously shifting along the same moving direction. This linear shift will induce a resistance to a sudden increase in flux penetration at the leading front because by nature only a gradual build-up of the flux density in the air gap is permitted. More specifically, as the primary coil set of the LIM moves, a new field penetrates into the reaction rail in the entry area, whereas the existing filed disappears at the exit area of the primary coil as shown below in Fig. 1 .

We should note that when the speed is higher, the air-gap flux is more unbalanced. Because the mutual flux between the primary and the secondary is decreased by the end effect, we can see that the equivalence of the end effect is a reduction force, which is a function of speed. As we know that most functions can be described in Taylor series reasonably, we assume that the end effect can be regarded as an external force which can be expressed as $\sum_{n=0}^{\infty} b_{n}^{\prime} v_{r}^{n}+M_{e} \dot{v}_{r}$. In the paper, we will truncate the series into the first three terms. 

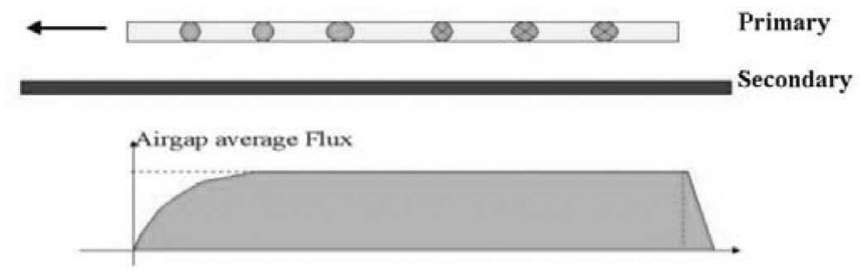

Fig. 1. Air-gap average flux distribution due to end effect.

As a result, for an LIM, the end effect plus the load force and the friction effect can be represented as a function of the speed $v_{r}$, which can normally be expressed by the following form:

$$
\begin{aligned}
F_{L}+F_{r}= & {\left[\left(\sum_{n=0}^{2} b_{n}^{\prime} v_{r}^{n}+M_{e} \dot{v}_{r}\right)+\bar{F}_{L}\right] } \\
& +\left[\zeta_{0} l+\zeta_{1}\left(v_{r}-\frac{\left|v_{r}\right|}{n\left(v_{r}\right)} l\right)+\zeta_{2} v_{r}\right] \\
= & {\left[M_{e} \dot{v}_{r}+b_{0}^{\prime}+b_{1}^{\prime} v_{r}+b_{2}^{\prime} v_{r}^{2}\right]+\left[M_{L}^{\prime} \dot{v}_{r}+b_{L 0}\right.} \\
& \left.+b_{L 1} v_{r}+b_{L 2} v_{r}^{2}\right]+\left[\zeta_{0} l+\left(\zeta_{1}+\zeta_{2}\right) v_{r}-\zeta_{1} \frac{\left|v_{r}\right|}{n\left(v_{r}\right)} l\right] \\
= & M_{L} \dot{v}_{r}+b o+b 1 v_{r}+b 2 v_{r}^{2}+\zeta_{0} l-\zeta_{1} \frac{\left|v_{r}\right|}{n\left(v_{r}\right)} l
\end{aligned}
$$

with

$$
\begin{aligned}
M_{L} & =M_{e}+M_{L}^{\prime L} \\
b_{0} & =b_{0}^{\prime+b_{L 0}} \\
b_{1} & =b_{1}^{\prime+b_{L 1}}+\zeta_{1}+\zeta_{2} \\
b_{2} & =b_{2}^{\prime+b_{L 2}}
\end{aligned}
$$

where $F_{L}$ is denoted as the mechanical payload accounting for the end effect and can be expressed in a compact form as $F_{L}=\Theta_{L}^{T} V_{r}$ with the unknown constant parameters $\Theta_{L}=\left[\begin{array}{llll}M_{L} & b_{0} & b_{1} & b_{2}\end{array}\right]^{T}$ and a known function vector $V_{r}=\left[\begin{array}{llll}\dot{v}_{r} & v_{r}^{0} & v_{r}^{1} & v_{r}^{2}\end{array}\right]^{T}$.

To proceed further, we introduce an additional assumptions as shown below:

Assumption 4: $x_{2}=\lambda_{q}^{2}+\lambda_{d}^{2}<0$

and then further simplify the dynamics shown in (1) by introducing a nonlinear coordinate transformation given as follows:

$$
\begin{aligned}
& x_{1}=i_{q}^{2}+i_{d}^{2} \\
& x_{2}=\lambda_{q}^{2}+\lambda_{d}^{2} \\
& x_{3}=i_{q} \lambda_{q}+i_{d} \lambda_{d} \\
& x_{4}=i_{q} \lambda_{d}-i_{d} \lambda_{q} \\
& x_{5}=v_{r} .
\end{aligned}
$$

Remark: The transformation tries to redefine the secondary flux norm, the electric force, and the primary speed as individual variables $x_{2}, x_{4}$, and $x_{5}$, respectively, and certainly the nonlinear transformation is nonunique.
Initially, let the stator voltage inputs be chosen as $c V_{d s}=$ $\frac{-\lambda_{q}}{\sqrt{\lambda_{d}^{2}+\lambda_{q}^{2}}} V$ and $c V_{q s}=\frac{\lambda_{d}}{\sqrt{\lambda_{d}^{2}+\lambda_{q}^{2}}} V$ [13]

where $V$ is an exogenous function to be defined later. Given such transformation and the chosen input, then the dynamical equations shown in (1) can thus be converted into the following dynamic model:

$$
\begin{aligned}
\dot{x}_{1} & =-2 a_{1} x_{1}+2 a_{2} x_{3}+2 x_{4} V / \sqrt{x_{2}} \\
\dot{x}_{2} & =-2 a_{4} x_{2}+2 a_{3} x_{3} \\
\dot{x}_{3} & =a_{3} x_{1}+a_{2} x_{2}-\left(a_{1}+a_{4}\right) x_{3}+p x_{5} x_{4} \\
\dot{x}_{4} & =-p x_{5} x_{3}-\beta p x_{5} x_{2}-\left(a_{1}+a_{4}\right) x_{4}+V \\
M \dot{x}_{5} & =K_{f} x_{4}-\sum_{n=0}^{2} b_{n} x_{5}^{n}-\zeta_{0} l+\zeta_{1} \frac{\left|x_{5}\right|}{n\left(x_{5}\right)} l
\end{aligned}
$$

where $M=M_{m}+M_{L}$. To control the system (6), we develop the position controller to achieve the goal of position tracking, namely, $p_{r}=\int_{0}^{t} x_{5} d t \rightarrow p_{d}$ as $t \rightarrow \infty$ will be explained in the following section.

\section{B. Flux Observer Design and Analysis}

Under our assumptions, all the states are measurable except the secondary flux. Therefore, we have to build a set of observers to estimate the secondary flux. The observers then can be designed as follows:

$$
\begin{aligned}
& \dot{\hat{\lambda}}_{q}=a_{3} i_{q}-a_{4} \hat{\lambda}_{q}+p v_{r} \hat{\lambda}_{d} \\
& \dot{\hat{\lambda}}_{d}=a_{3} i_{d}-a_{4} \hat{\lambda}_{d}-p v_{r} \hat{\lambda}_{q} .
\end{aligned}
$$

Theroem 1: [13] If the dynamical equations are described as in system (6) with all the states being measurable except the secondary flux. Moreover, all the parameters are assumed known beforehand. Then, the flux observers designed can guarantee that both $\hat{\lambda}_{d}-\lambda_{d} \rightarrow 0$ and $\hat{\lambda}_{q}-\lambda_{q} \rightarrow 0$ as $t \rightarrow \infty$.

\section{Two Nonlinear Observer Designs for Friction Estimation}

In this paper, we consider the frictional effect represented by a LuGre model as described by (3) and (4). But we know that the friction state $l$ is not measurable. In order to handle different nonlinearities due to $l$ which are present in the system dynamics, we employ two nonlinear observers to estimate the unmeasurable state $l$ and replace $l$ with its estimates $\hat{l}_{0}$ and $\hat{l}_{1}$ [16] and [17], of which the dynamics are respectively given by

$$
\begin{aligned}
& \frac{d \hat{l}_{0}}{d t}=x_{5}-\frac{\left|x_{5}\right|}{n\left(x_{5}\right)} \hat{l}_{0}+\eta_{0} \\
& \frac{d \hat{l}_{1}}{d t}=x_{5}-\frac{\left|x_{5}\right|}{n\left(x_{5}\right)} \hat{l}_{1}+\eta_{1}
\end{aligned}
$$

where $\eta_{0}, \eta_{1}$ are compensation terms yet to be determined in later design. The corresponding observation errors can be 
computed as

$$
\begin{aligned}
& \frac{d \tilde{l}_{0}}{d t}=-\frac{\left|x_{5}\right|}{n\left(x_{5}\right)} \tilde{l}_{0}-\eta_{0} \\
& \frac{d \tilde{l}_{1}}{d t}=-\frac{\left|x_{5}\right|}{n\left(x_{5}\right)} \tilde{l}_{1}-\eta_{1}
\end{aligned}
$$

where $\tilde{l}_{0}=l-\hat{l}_{0}$ and $\tilde{l}_{1}=l-\hat{l}_{1}$ are estimation errors.

\section{Nonlinear Adaptive Position Controller With Friction Compensation Design}

Now, we introduce another state

$$
x_{6}=p_{r}
$$

to facilitate investigation of the development of a position controller. Then, we define the tracking errors as follows:

$$
e_{p}=p_{r}-p_{d}=e_{6}
$$

Normally, while the position tracking error is driven to zero, the speed is also regulated to zero. Thus, we naturally define a joint error signal $S$ as follows:

$$
S=\dot{e}_{p}+a e_{p}=e_{5}+a e_{6}
$$

where $a$ is a positive scalar gain. The resulting error dynamics are derived as follows:

$$
\begin{aligned}
M \dot{S}= & K_{f} x_{4}-\sum_{n=0}^{2} b_{n} x_{5}^{n}-M\left(\dot{v}_{d}-a e_{5}\right)-\zeta_{0} l+\zeta_{1} \frac{\left|x_{5}\right|}{n\left(x_{5}\right)} l \\
= & K_{f} x_{4}-\sum_{n=0}^{2} b_{n} x_{5}^{n}-M\left(\dot{v}_{d}-a e_{5}\right)-\zeta_{0}\left(\tilde{l}_{0}+\hat{l}_{0}\right) \\
& +\zeta_{1} \frac{\left|x_{5}\right|}{n\left(x_{5}\right)}\left(\tilde{l}_{1}+\hat{l}_{1}\right) .
\end{aligned}
$$

Based on this equation, we will propose a position tracking controller, and the following theorem summarizes the design procedure and the resulting control effect.

Theorem 2: Consider a linear induction motor whose dynamics are governed by system (6) under the assumptions (A.1)(A.4). Given friction observer (7) and a smooth desired position trajectory $p_{d}$ with $p_{d}, \dot{p}_{d}, \ddot{p}_{d}$ and $\ddot{p}_{d}$ being all bounded, then the following control input can achieve the control objective $p_{r} \rightarrow p_{d}$ as $t \rightarrow \infty$ (i.e., $x_{6}=p_{r}$ will follow $p_{d}$ asymptotically) with the control input

$$
V_{q s}=\frac{\lambda_{d}}{\sqrt{\lambda_{q}^{2}+\lambda_{d}^{2}}} \frac{V}{c} \quad V_{d s}=\frac{-\lambda_{q}}{\sqrt{\lambda_{q}^{2}+\lambda_{d}^{2}}} \frac{V}{c}
$$

and

$$
\begin{aligned}
V= & \frac{1}{\sqrt{x_{2}}}\left[\left(a_{1}+a_{4}\right) x_{4}+\beta p x_{2} x_{5}+p x_{3} x_{5}\right. \\
& \left.+\dot{x}_{4 d}-\rho_{2} e_{4}-K_{f} S\right]
\end{aligned}
$$

where

$x_{4 d}=\frac{1}{K_{f}}\left[\sum_{n=0}^{2} \hat{b}_{n} x_{5}^{n}+\hat{M}\left(\dot{v}_{d}-a e_{5}\right)+\hat{\zeta}_{0} \hat{l}_{0}-\hat{\zeta}_{1} \frac{\left|x_{5}\right|}{n\left(x_{5}\right)} \hat{l}_{1}-\rho_{1} S\right]$ with $\rho_{1}, \rho_{2}>0$, and $e_{5}=x_{5}-v_{d}, e_{4}=x_{4}-x_{4 d}$, and the parameter adaptation laws

$$
\begin{aligned}
& \dot{\hat{b}}_{0}=-S, \dot{\hat{b}}_{1}=-S x_{5} \\
& \dot{\hat{b}}_{2}=-S x_{5}^{2} \\
& \dot{\hat{M}}=-S\left(\dot{v}_{d}-a e_{5}\right) \\
& \dot{\hat{\zeta}}_{0}=-S \hat{l}_{0} \\
& \dot{\hat{\zeta}}_{1}=-S \frac{\left|x_{5}\right|}{n\left(x_{5}\right)} \hat{l}_{1}
\end{aligned}
$$

subject to the design of the compensation terms for the friction observer as follows:

$$
\eta_{0}=-S \quad \eta_{1}=-\frac{\left|x_{5}\right|}{n\left(x_{5}\right)} S
$$

while keeping all the internal signals bounded.

Proof: In order to show the boundedness of all the parameter estimates and the tracking errors $e_{4}, e_{5}$, we choose a Lyapunovlike function $V_{e}$ as shown by:

$V_{e}=\frac{1}{2}\left[M S^{2}+e_{4}^{2}+\tilde{b}_{0}^{2}+\tilde{b}_{1}^{2}+\tilde{b}_{2}^{2}+\tilde{M}^{2}+\tilde{\zeta}_{0}^{2}+\tilde{\zeta}_{1}^{2}+\tilde{l}_{0}^{2}+\tilde{l}_{1}^{2}\right]$

According to the proposed adaptive laws as

$$
\begin{aligned}
& \dot{\hat{b}}_{0}=-S \\
& \dot{\hat{b}}_{1}=-S x_{5} \\
& \dot{\hat{b}}_{2}=-S x_{5}^{2} \\
& \dot{\hat{M}}=-S\left(\dot{v}_{d}-a e_{5}\right) \\
& \dot{\hat{\zeta}}_{0}=-S \hat{l}_{0} \\
& \dot{\hat{\zeta}}_{1}=-S \frac{\left|x_{5}\right|}{n\left(x_{5}\right)} \hat{l}_{1}
\end{aligned}
$$

and the suggested compensation terms for the friction observer as:

$$
\eta_{0}=-S \quad \eta_{1}=-\frac{\left|x_{5}\right|}{n\left(x_{5}\right)} S .
$$

If one designs the auxiliary signal $x_{4 d}$ as

$x_{4 d}=\frac{1}{K_{f}}\left[\sum_{n=0}^{2} \hat{b}_{n} x_{5}^{n}+\hat{M}\left(\dot{v}_{d}-a e_{5}\right)+\hat{\zeta}_{0} \hat{l}_{0}-\hat{\zeta}_{1} \frac{\left|x_{5}\right|}{n\left(x_{5}\right)} \hat{l}_{1}-\rho_{1} S\right]$

then the time derivative of the function $V_{e}$ becomes

$$
\begin{aligned}
\dot{V}_{e}= & e_{4}\left[-\left(a_{1}+a_{4}\right) x_{4}-\beta p x_{2} x_{5}-p x_{3} x_{5}+\sqrt{x_{2}} V\right. \\
& \left.-\dot{x}_{4 d}+K_{f} S\right]-\rho_{1} S^{2}-\zeta_{0} \frac{\left|x_{5}\right|}{n\left(x_{5}\right)} \tilde{l}_{0}^{2}-\zeta_{1} \frac{\left|x_{5}\right|}{n\left(x_{5}\right)} \tilde{l}_{1}^{2} .
\end{aligned}
$$

Now, by hypothesis the actual input is designed as $V=\frac{1}{\sqrt{x_{2}}}\left[\left(a_{1}+a_{4}\right) x_{4}+\beta p x_{2} x_{5}+p x_{3} x_{5}+\dot{x}_{4 d}-\rho_{2} e_{4}-K_{f} S\right]$ 
which apparently leads to the result that

$$
\dot{V}_{e}=-\rho_{1} S^{2}-\rho_{2} e_{4}^{2}-\zeta_{0} \frac{\left|x_{5}\right|}{n\left(x_{5}\right)} \tilde{l}_{0}^{2}-\zeta_{1} \frac{\left|x_{5}\right|}{n\left(x_{5}\right)} \tilde{l}_{1}^{2} \leq 0
$$

where $\rho_{1}, \rho_{2}>0, \zeta_{0}, \zeta_{1}$ are positive constants, and the friction characteristic function $n\left(x_{5}\right)$ is chosen to be a positive function, which readily implies boundedness of all parameter estimates as well as of both signals $x_{4}$ and $x_{5}$. The reason is as follows: Since $\dot{V}_{e}$ in (11) is nonpositive, we conclude that all the error signals in $V_{e}$ and in particular, $x_{5}$ and $x_{4 d}$ are bounded, which in turn implies that $x_{4}$ and hence, [from system (6)] are both bounded. This further implies that the estimation errors $\tilde{l}_{0}, \tilde{l}_{1} \in$ $L_{\infty}$, the parametric errors $\tilde{\zeta}_{0}, \tilde{\zeta}_{1} \in L_{\infty}$, and hence, the parameter estimates $\hat{\zeta}_{0}, \hat{\zeta}_{1} \in L_{\infty}$. From the friction dynamics in (3) and the boundedness of speed $x_{5}$, boundedness of the friction state $l$ is clear, which then implies the observer states $\hat{l}_{0}, \hat{l}_{1}$ are bounded. Now, we are ready to show that all the internal signals are kept bounded. By using a fact that if $I_{s}$ is bounded, then all signals $x_{i}, \lim _{i=1}, ., 5$. are then guaranteed to be bounded.

By the power formula, $P_{s}=K_{f} x_{4} x_{5}=3 V_{s} I_{s}$ can be shown bounded from the above reasoning. We now want to show that $I_{s}$ will be bounded via argument of contradiction: say, $I_{s}$ eventually grows unbounded, then $V_{s}$ and hence, $V$ will diminish eventually; however, if $I_{s}$ does grow unbound, then it implies that $V$ will tend to $p x_{5} x_{3} / \sqrt{x_{2}}$ eventually. But, from the dynamics of $x_{2}$ in (6), we have $x_{2}$ and $x_{3}$ grow at the same rate, which readily says that $V$ form the hypothesis will also grow unbounded. This obviously leads to a contradiction and therefore, $I_{s}$ is bounded.

Furthermore, we can show that $\dot{x}_{4 d}$ is bounded, and hence, $\dot{e}_{4}$ and $\dot{S}$ are also bounded, which implies the convergence of $e_{4}$ and $S$ due to Barbalat's Lemma. Therefore, the control scheme with the properly designed input $V$ from the hypothesis will drive the output $p_{r}$ to the desired $p_{d}$ asymptotically.

\section{E. Consideration of Uncertain Inductance}

From the previous LIM dynamics, the parameters $a_{1}, \beta, c$ and $K_{f}$ depend on the mutual inductance, but as we know the mutual inductance is hard to identify due to its intricate structure and undesirable end effect. In particular,

$$
\begin{aligned}
a_{1} & =\frac{R_{s} L_{r}+R_{r} L_{m}^{2} / L_{r}}{L_{s} L_{r}-L_{m}^{2}}=a_{10}+\alpha, \\
c & =\frac{L_{r}}{L_{s} L_{r}-L_{m}^{2}}=c_{0}+\sigma
\end{aligned}
$$

where $\alpha$ and $\sigma$ are uncertainty terms of $a_{1}$ and $c$, respectively. We rewrite the dynamic (6) as follows:

$$
\begin{aligned}
\dot{x}_{1}= & -2\left(a_{10}+\alpha\right) x_{1}+2 a_{2} x_{3}+\frac{2\left(c_{0}+\sigma\right) x_{4}}{\sqrt{x_{2}}} V \\
\dot{x}_{2}= & -2 a_{4} x_{2}+2 a_{3} x_{3} \\
\dot{x}_{3}= & a_{3} x_{1}+a_{2} x_{2}-\left(a_{10}+\alpha+a_{4}\right) x_{3}+p x_{5} x_{4} \\
\dot{x}_{4}= & -p x_{5} x_{3}-\beta p x_{5} x_{2}-\left(a_{10}+\alpha+a_{4}\right) x_{4} \\
& +\left(c_{0}+\sigma\right) \sqrt{x_{2}} V
\end{aligned}
$$

$$
\begin{aligned}
\frac{M}{K_{f}} \dot{x}_{5} & =x_{4}-\sum_{n=0}^{2} \frac{b_{n}}{K_{f}} x_{5}^{n}-\frac{\zeta_{0}}{K_{f}} l+\frac{\zeta_{1}}{K_{f}} \frac{\left|x_{5}\right|}{n\left(x_{5}\right)} l \\
\dot{x}_{6} & =x_{5}
\end{aligned}
$$

and design the control input

$$
V_{q s}=\frac{\lambda_{d}}{\sqrt{\lambda_{q}^{2}+\lambda_{d}^{2}}} V \quad V_{d s}=\frac{-\lambda_{q}}{\sqrt{\lambda_{q}^{2}+\lambda_{d}^{2}}} V
$$

To facilitate subsequent investigation, we define several variables as follows:

$$
\begin{aligned}
\tilde{\alpha} & =\alpha-\hat{\alpha} \\
\tilde{\beta} & =\beta-\hat{\beta} \\
d_{n} & =\frac{b_{n}}{K_{f}} \\
H & =\frac{M}{K_{f}} \\
\xi_{0} & =\frac{\xi_{0}}{K_{f}} \\
\xi_{1} & =\frac{\xi_{1}}{K_{f}}
\end{aligned}
$$

where $\hat{\alpha}$ is the estimate of $\alpha, \hat{\beta}$ is the estimate of $\beta$.

In order to show the boundedness of all the parameter estimates and the tracking errors $e_{4}, S$, we choose a Lyapunov like function $V_{e}$ as shown by

$$
\begin{aligned}
V_{e}=\frac{1}{2}\left[H S^{2}+\right. & e_{4}^{2}+\tilde{d}_{0}^{2}+\tilde{d}_{1}^{2}+\tilde{d}_{2}^{2} \\
& \left.+\tilde{H}^{2}+\tilde{\xi}_{0}^{2}+\tilde{\xi}_{1}^{2}+\tilde{l}_{0}^{2}+\tilde{l}_{1}^{2}+\tilde{\alpha}^{2}+\tilde{\beta}^{2}\right]
\end{aligned}
$$

where

$$
\begin{aligned}
& \tilde{H}=\hat{H}-H \\
& \tilde{\xi}_{0}=\hat{\xi}_{0}-\xi_{0} \\
& \tilde{\xi}_{1}=\hat{\xi}_{1}-\xi_{1} \\
& \tilde{d}_{0}=\hat{d}_{0}-d_{0} \\
& \tilde{d}_{1}=\hat{d}_{1}-d_{1} \\
& \tilde{d}_{2}=\hat{d}_{2}-d_{2} .
\end{aligned}
$$

If we employ friction observer (7) and design the parameter adaptive laws as

$$
\begin{aligned}
& \dot{\hat{d}}_{0}=-S \\
& \dot{\hat{d}}_{1}=-S x_{5} \\
& \dot{\hat{d}}_{2}=-S x_{5}^{2} \\
& \dot{\hat{H}}=-S\left(\dot{v}_{d}+a e_{5}\right) \\
& \dot{\hat{\alpha}}=-e_{4} x_{4} \dot{\hat{\beta}}=-e_{4} p x_{5} x_{2}
\end{aligned}
$$




$$
\begin{aligned}
& \dot{\hat{\xi}}_{0}=-S \hat{l}_{0} \\
& \dot{\hat{\xi}}_{1}=-S \frac{\left|x_{5}\right|}{n\left(x_{5}\right)} \hat{l}_{1}
\end{aligned}
$$

with the friction observer compensation terms being defined as

$$
\eta_{0}=-S \quad \eta_{1}=-\frac{\left|x_{5}\right|}{n\left(x_{5}\right)} S
$$

together with the proper design of

$x_{4 d}=\left[\sum_{n=0}^{2} \hat{d}_{n} x_{5}^{n}+\hat{H}\left(\dot{v}_{d}-a e_{5}\right)+\hat{\xi}_{0} \hat{l}_{0}-\hat{\xi}_{1} \frac{\left|x_{5}\right|}{n\left(x_{5}\right)} \hat{l}_{1}-\rho_{1} S\right]$

then the time derivative of the Lyapunov-like function $\dot{V}_{e}$ becomes

$$
\begin{aligned}
\dot{V}_{e}=-\rho_{1} S^{2}+e_{4}\left[g(x)+\left(c_{0}+\sigma\right)\right. & \left.\sqrt{x_{2}} V\right] \\
& \quad-\xi_{0} \frac{\left|x_{5}\right|}{n\left(x_{5}\right)} \tilde{l}_{0}^{2}-\xi_{1} \frac{\left|x_{5}\right|}{n\left(x_{5}\right)} \tilde{l}_{1}^{2}
\end{aligned}
$$

where $g(x)=-p x_{5} x_{3}-\beta p x_{5} x_{2}-\left(a_{10}+\alpha+a_{4}\right) x_{4}-\dot{x}_{4 d}$.

After we substitute the properly designed input $V$ as

$$
V=\frac{1}{c_{0} \sqrt{x_{2}}}\left\{-g(x)-\eta \operatorname{sgn}\left(e_{4}\right)\right\}
$$

where $\operatorname{sgn}(\cdot)$ is the sign function, then the time derivative $\dot{V}_{e}$ can be reexpressed as

$$
\begin{aligned}
\dot{V}_{e}= & -\rho_{1} S^{2}-\xi_{0} \frac{\left|x_{5}\right|}{n\left(x_{5}\right)} \tilde{l}_{0}^{2}-\xi_{1} \frac{\left|x_{5}\right|}{n\left(x_{5}\right)} \tilde{l}_{1}^{2} \\
& -\left(\frac{c_{0}+\sigma}{c_{0}}\right) e_{4}\left[\eta \operatorname{sgn}\left(e_{4}\right)+\left(\frac{c_{0}}{c_{0}+\sigma}\right) g(x, S)\right\} \\
& \leq-\rho_{1} S^{2}-\xi_{0} \frac{\left|x_{5}\right|}{n\left(x_{5}\right)} \tilde{l}_{0}^{2}-\xi_{1} \frac{\left|x_{5}\right|}{n\left(x_{5}\right)} \tilde{l}_{1}^{2} \\
& -\left(\frac{c_{0}+\sigma}{c_{0}}\right)\left[\eta-\left(\frac{c_{0}}{c_{0}+\sigma}\right)|g(x, S)|\right\}\left|e_{4}\right| .
\end{aligned}
$$

Now, if $\eta$ is chosen to satisfy $\eta \geq|g(x)|+k$ for some $k>0$, then we have

$$
\dot{V}_{e} \leq-\rho_{1} S^{2}-\xi_{0} \frac{\left|x_{5}\right|}{n\left(x_{5}\right)} \tilde{l}_{0}^{2}-\xi_{1} \frac{\left|x_{5}\right|}{n\left(x_{5}\right)} \tilde{l}_{1}^{2}-\rho_{2}\left|e_{4}\right|
$$

for some $\rho_{2}>0$, which again implies boundedness of all internal signals and convergence of the position tracking error by the argument similar to that in Section III and IV.

\section{AdAPTIVE BACKSTEPPing Controller Design}

Remark: In the previous section, we have proposed an adaptive controller for the LIMs, which will require acceleration signals of the motor. Although this signal can be obtained through numerical differencing and digital filtering, it is more susceptible to noise. In order to avoid such problem, we propose the following nonlinear backstepping position controller without need of acceleration signal in this section.

Theorem 3: Consider a linear induction motor whose dynamics are governed by system (6) under the assumption (A.1)(A.4). Given a friction observer (7) a smooth desired position trajectory $p_{d}$ with $p_{d}, \dot{p}_{d}, \ddot{p}_{d}$ and $\ddot{p}_{d}$ being all bounded, then the following control input can achieve the control objective $p_{r} \rightarrow p_{d}$ as (i.e., $x_{6}=p_{r}$ will follow $p_{d}$ asymptotically) with the control input

$$
V_{q s}=\frac{\lambda_{d}}{\sqrt{\lambda_{q}^{2}+\lambda_{d}^{2}}} \frac{V}{c} \quad V_{d s}=\frac{-\lambda_{q}}{\sqrt{\lambda_{q}^{2}+\lambda_{d}^{2}}} \frac{V}{c},
$$

and

$$
V=\frac{1}{\sqrt{x_{2}}}\left[g_{1}(x)+\hat{\Theta}_{1}^{T} V_{r 1}-K_{f} z_{1}-\rho_{2} z_{2}\right]
$$

with adaptation law given as

$$
\begin{aligned}
& \dot{\hat{\Theta}}=-\Gamma_{1} z_{1} V_{r} \\
& \dot{\hat{\Theta}}_{1}=-\Gamma_{2} z_{2} V_{r 1} \\
& \dot{\hat{\xi}}_{0}=-z_{1} \hat{l}_{0} \\
& \dot{\hat{\xi}}_{1}=-z_{1} \frac{\left|x_{5}\right|}{n\left(x_{5}\right)} \hat{l}_{1}
\end{aligned}
$$

and compensation terms of the friction observer specified as

$$
\eta_{0}=-z_{1} \quad \eta_{1}=-\frac{\left|x_{5}\right|}{n\left(x_{5}\right)} z_{1}
$$

where $\Theta=\left[\begin{array}{llll}M & b_{0} & b_{1} & b_{2}\end{array}\right]^{T}, V_{r}=\left[\begin{array}{llll}\dot{v}_{r} & v_{r}^{0} & v_{r}^{1} & v_{r}^{2}\end{array}\right]^{T}$,

$$
\Gamma_{1}, \Gamma_{2}>0, \quad z_{1}=S, \quad z_{2}=x_{4}-\alpha_{1},
$$

$\alpha_{1}=-\rho_{1} M z_{1}+\frac{1}{K_{f}} \hat{\Theta}^{T} V_{r}-\frac{a}{K_{f}} M e_{5}+\hat{\xi}_{0} \hat{l}_{0}-\hat{\xi}_{1} \frac{\left|x_{5}\right|}{n\left(x_{5}\right)} \hat{l}_{1}$

for some $\rho_{1}, \rho_{2}>0$, and

$$
\begin{aligned}
g_{1}(x)= & p x_{3} x_{5}+\beta x_{2} x_{5}+\left(a_{1}+a_{4}-a\right) x_{4} \\
& -\rho_{1}\left(K_{f} x_{4}+\kappa J e_{6}\right), \\
\Theta_{1}^{T} V_{r 1}= & \left(\rho_{1}+\frac{a}{K_{f}}\right) \Theta^{T} V_{r}+\frac{1}{K_{f}} \Theta^{T} \dot{V}_{r} \\
= & \left(\rho_{1}+\frac{a}{K_{f}}\right) \Theta^{T} V_{r}+\frac{1}{K_{f}} \Theta^{\prime T} V_{r}^{\prime}
\end{aligned}
$$

with the parameter vector $\Theta^{\prime}$ as well as the known function vector $V_{r}^{\prime}$ satisfying

$$
\Theta^{T} \dot{V}_{r}=\Theta^{\prime T} V_{r}^{\prime}
$$

keeping all the internal system signals bounded.

Proof:

Step 1. Choose a different stabilizing function $\alpha_{1}$ as follows:

$\alpha_{1}=-\rho_{1} M z_{1}+\frac{1}{K_{f}} \hat{\Theta}^{T} V_{r}-\frac{a}{K_{f}} M e_{5}+\hat{\xi}_{0} \hat{l}_{0}-\hat{\xi}_{1} \frac{\left|x_{5}\right|}{n\left(x_{5}\right)} \hat{l}_{1}$

where $\hat{\Theta}$ denotes the online parameter estimate. Also, redefine the new error variables $z_{1}=S, \mathrm{z}_{2}=x_{4}-\alpha_{1}$.

Evaluate the time derivative of the Lyapunov-like function shown by

$$
V_{1}=\frac{1}{2} M z_{1}^{2}+\frac{1}{2 \Gamma_{1}} \tilde{\Theta}^{T} \tilde{\Theta}+\frac{1}{2} \tilde{\xi}_{0}^{2}+\frac{1}{2} \tilde{\xi}_{1}^{2}+\frac{1}{2} \tilde{l}_{0}^{2}+\frac{1}{2} \tilde{l}_{1}^{2}
$$


along the solution trajectories to obtain

$$
\begin{aligned}
\dot{V}_{1}= & -\rho_{1} K_{f} M z_{1}^{2}+K_{f} z_{1} z_{2} \\
& +\tilde{\Theta}^{T}\left(\frac{1}{\Gamma_{1}} \dot{\tilde{\Theta}}+z_{1} V_{r}\right)+\tilde{\xi}_{0}\left(\dot{\tilde{\xi}}_{0}-z_{1} \hat{l}_{0}\right) \\
& +\tilde{\xi}_{1}\left(\dot{\tilde{\xi}}_{1}-z_{1} \frac{\left|x_{5}\right|}{n\left(x_{5}\right)} \hat{l}_{1}\right)-\xi_{0} \tilde{l}_{0}\left(\eta_{0}+z_{1}\right) \\
& +\xi_{1} \tilde{l}_{1}\left(\eta_{1}+\frac{\left|x_{5}\right|}{n\left(x_{5}\right)} z_{1}\right) \\
& -\xi_{0} \frac{\left|x_{5}\right|}{n\left(x_{5}\right)} \tilde{l}_{0}^{2}-\xi_{1} \frac{\left|x_{5}\right|}{n\left(x_{5}\right)} \tilde{l}_{1}^{2}
\end{aligned}
$$

which motivates us to choose the adaptation law as

$$
\begin{aligned}
& \dot{\tilde{\Theta}}=\dot{\hat{\Theta}}=-\Gamma_{1} z_{1} V_{r} \\
& \dot{\hat{\xi}}_{0}=-z_{1} \hat{l}_{0} \\
& \dot{\hat{\xi}}_{1}=-z_{1} \frac{\left|x_{5}\right|}{n\left(x_{5}\right)} \hat{l}_{1} \\
& \eta_{0}=-z_{1} \\
& \eta_{1}=-\frac{\left|x_{5}\right|}{n\left(x_{5}\right)} z_{1}
\end{aligned}
$$

for some proper positive adaptation gain $\Gamma_{1}$. As a result, (16) can be slightly simplified as:

$$
\dot{V}_{1}=-\rho_{1} K_{f} M z_{1}^{2}+K_{f} z_{1} z_{2}-\xi_{0} \frac{\left|x_{5}\right|}{n\left(x_{5}\right)} \tilde{l}_{0}^{2}-\xi_{1} \frac{\left|x_{5}\right|}{n\left(x_{5}\right)} \tilde{l}_{1}^{2} .
$$

Step 2. The time derivative of $z_{2}$ is now expressed as

$$
\dot{z}_{2}=\dot{x}_{4}-\dot{\alpha}_{1}=-g_{1}(x)-\Theta_{1}^{T} V_{r 1}+\sqrt{x_{2}} V
$$

where the functions involved above are as previously defined. Thus, we need to select a Lyapunov-like function and design $V$ to render its time derivative nonpositive. We want to apply the augmented Lyapunov-like function candidate as

$$
V_{2}=V_{1}+\frac{1}{2} z_{2}^{2}
$$

whose time derivative is found to be

$$
\begin{gathered}
\dot{V}_{2}=-\rho_{1} K_{f} M z_{2}^{2}+K_{f} z_{1} z_{2}+z_{2}\left[-g_{1}(x)-\Theta_{1}^{T} V_{r 1}+\sqrt{x_{2}} V\right] \\
-\xi_{0} \frac{\left|x_{5}\right|}{n\left(x_{5}\right)} \tilde{l}_{0}^{2}-\xi_{1} \frac{\left|x_{5}\right|}{n\left(x_{5}\right)} \tilde{l}_{1}^{2} .
\end{gathered}
$$

The control law $V$ should be able to cancel the indefinite term in (21). On the other hand, to deal with the unknown parameters $\Theta_{1}$, we will try to employ the current estimates $\hat{\Theta}_{1}$, i.e.,

$$
V=\frac{1}{\sqrt{x_{2}}}\left[g_{1}(x)+\hat{\Theta}_{1}^{T} V_{r 1}-K_{f} z_{1}-\rho_{2} z_{2}\right] .
$$

From this resulting derivative

$$
\begin{aligned}
\dot{V}_{2}=-\rho_{1} K_{f} M z_{1}^{2}+z_{2} \hat{\Theta}_{1} & V_{r 1}-\rho_{2} z_{2}^{2} \\
& -\xi_{0} \frac{\left|x_{5}\right|}{n\left(x_{5}\right)} \tilde{l}_{0}^{2}-\xi_{1} \frac{\left|x_{5}\right|}{n\left(x_{5}\right)} \tilde{l}_{1}^{2}
\end{aligned}
$$

in order to cancel the last term in (19), we modify the Lyapunovlike function as follows

$$
V_{3}=V_{2}+\frac{1}{2} z_{2}^{2}+\frac{1}{2} \tilde{\Theta}_{1}^{T} \tilde{\Theta}_{1}
$$

and the time derivative of $V_{3}$, hence, is

$$
\begin{aligned}
\dot{V}_{3}=-\rho_{1} K_{f} M z_{1}^{2}+\hat{\Theta}_{1}^{T}\left(z_{2} V_{r 1}\right. & \left.+\frac{1}{\Gamma_{2}} \dot{\tilde{\Theta}}_{1}\right) \\
& \quad-\xi_{0} \frac{\left|x_{5}\right|}{n\left(x_{5}\right)} \tilde{l}_{0}^{2}-\xi_{1} \frac{\left|x_{5}\right|}{n\left(x_{5}\right)} \tilde{l}_{1}^{2} .
\end{aligned}
$$

Now, the term with $\tilde{\Theta}_{1}$ can be eliminated completely with the update law

$$
\dot{\tilde{\Theta}}_{1}=\dot{\hat{\Theta}}_{1}=-\Gamma_{2} z_{2} V_{r 1}
$$

for some positive adaptation gain $\Gamma_{2}$,which thus yields

$$
\dot{V}_{3}=-\rho_{1} K_{f} M z_{1}^{2}-\rho_{2} z_{2}^{2}-\xi_{0} \frac{\left|x_{5}\right|}{n\left(x_{5}\right)} \tilde{l}_{0}^{2}-\xi_{1} \frac{\left|x_{5}\right|}{n\left(x_{5}\right)} \tilde{l}_{1}^{2}
$$

which guarantees boundedness of all parameter estimates $\hat{\Theta}$, $\hat{\Theta}_{1}$ and $z_{1}, z_{2}$, and $z_{1} \in L^{2} \cap L^{\infty}$. To show boundedness of the rest of states, we can rearrange the dynamical equations from system (6) as shown by

$$
\begin{aligned}
\dot{X}= & {\left[\begin{array}{l}
\dot{x}_{1} \\
\dot{x}_{2} \\
\dot{x}_{3}
\end{array}\right]=\left[\begin{array}{ccc}
-2 a_{1} & 0 & 2 a_{2} \\
0 & -2 a_{4} & 2 a_{3} \\
a_{3} & a_{2} & -\left(a_{1}+a_{4}\right)
\end{array}\right]\left[\begin{array}{l}
x_{1} \\
x_{2} \\
x_{3}
\end{array}\right] } \\
& +\left[\begin{array}{c}
\frac{2 x_{4}}{\sqrt{x_{2}}} V \\
0 \\
p x_{5} x_{4}
\end{array}\right] \\
= & A X+u
\end{aligned}
$$

where $A$ can be shown to be Hurwitz. After reviewing definitions of $x_{3}$ and $V$, repectively, we found that the first entry of $u$ will be bounded because $x_{2}$ grows no slower than $x_{3}$ if $x_{3}$ does grow unbounded [due to the second of (6)]. As a result, $u$ is apparently bounded, and hence, $X$ will be bounded. This then proves the boundedness of all the states. We note that $\dot{z}_{1}$ is also bounded, and hence, by Barbalat's lemma we can conclude that

$$
\lim _{l \rightarrow \infty} z_{1} \rightarrow 0, \text { i.e., } p_{r} \rightarrow p_{d} \text { as } t \rightarrow \infty \text {. }
$$

\section{Simulation Results}

In our simulation, the parameters of the linear induction motor we use are according to Table I. All the results will be demonstrated in the following sections.

\section{A. Results of Nonlinear Adaptive Controller}

In order to confirm the performance of the controller design in Section III. We proposed several kinds of desired position trajectories, including exponential function, sinusoidal function, etc. All the results are shown in Figs. 2 and 3. It is found that both the observed states and the estimated parameters are 
TABLE I

SPECIFICATIONS AND PARAMETERS OF THE MOTOR

\begin{tabular}{|ll|l|}
\hline \multicolumn{2}{|c|}{ Specification } & Parameters normal value \\
\hline 3 Phase (Y-connected) & $\mathrm{R}_{\mathrm{s}}=13.2 \Omega$ \\
Rated Power $\quad$ 1HP & $\mathrm{R}_{\mathrm{r}}=11.78 \Omega$ \\
Rated Air gap & $0.125 \mathrm{in}$ & $\mathrm{L}_{\mathrm{s}}=0.42 \mathrm{H}$ \\
Rated Current $\quad 5 \mathrm{~A}$ & $\mathrm{~L}_{\mathrm{r}}=0.42 \mathrm{H}$ \\
Rated Voltage $\quad 240 \mathrm{~V}$ & $\mathrm{~L}_{\mathrm{m}}=0.4 \mathrm{H}$ \\
Rated Poles & 4 & $\mathrm{M}=4.775 \mathrm{Kg}$ \\
Pitch & $46.5 \mathrm{~mm}$ & $\mathrm{~B}=53 \mathrm{Kg} / \mathrm{sec}$ \\
Secondary length $\quad 82 \mathrm{~cm}$ &
\end{tabular}

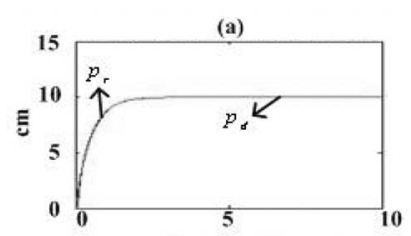

(b)

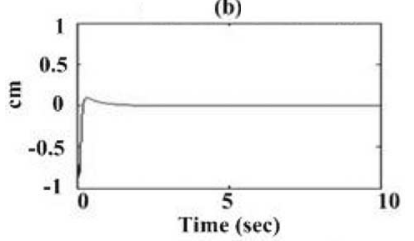

(c)

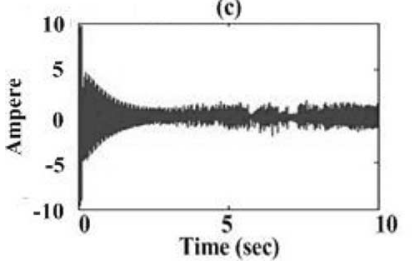

Fig. 2. Desired position trajectories, exponential function, sinusuidal function, $p_{d}=10\left(1-e^{-2 t}\right) \mathrm{cm}$
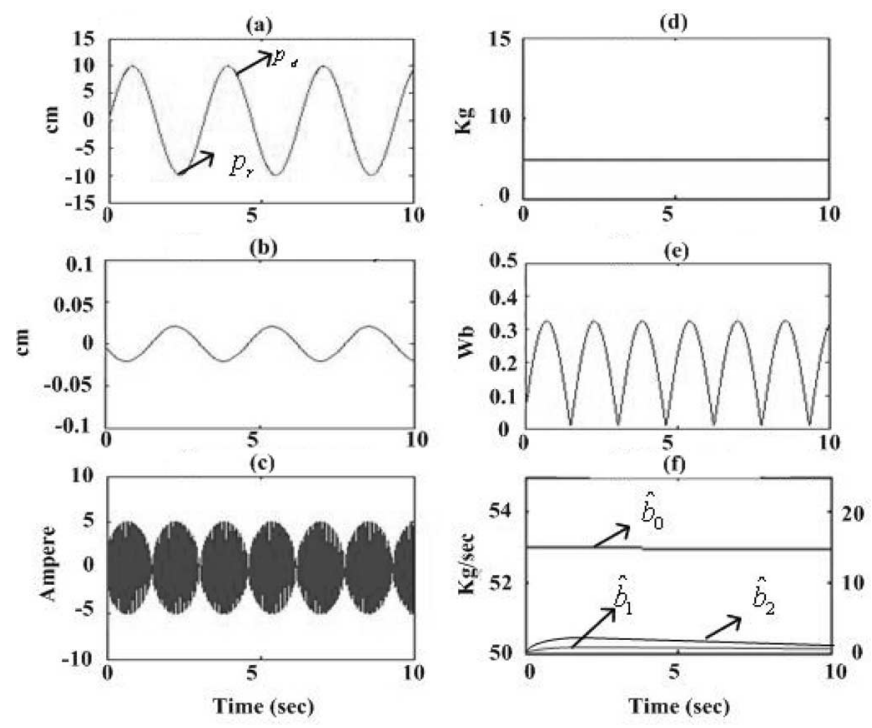

Fig. 3. $p_{d}=10 \sin (2 t) \mathrm{cm}$.

shown bounded, and the position tracking errors do converge. The control gains and initial values are chosen in Table II.
TABLE II

CONTROLler GAIN AND INITIAL VALUES

\begin{tabular}{|c|c|c|c|c|}
\hline \multicolumn{5}{|c|}{ Controller Gain } \\
\hline \multicolumn{2}{|c|}{$\rho_{1}$} & \multicolumn{2}{c|}{$\rho_{2}$} \\
\hline \multicolumn{2}{|c|}{200.0} & $\hat{\lambda}_{q}$ & others \\
\hline Initial value & $\hat{M}$ & $\hat{\lambda}_{d}$ & $\hat{n}$ & 0.0 \\
\hline$\hat{b}_{0}$ & $\hat{M}$ & 0.0001 & 0.0001 & 0.0 \\
\hline 53.0 & 4.773 & 0.00
\end{tabular}

(a)
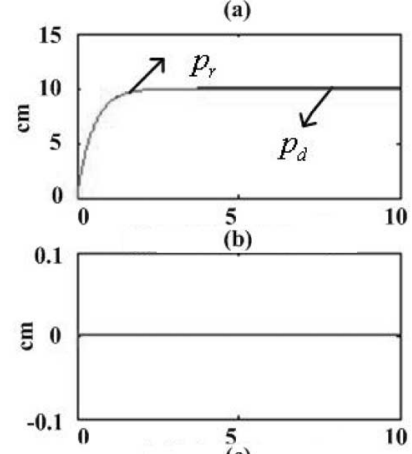

(c)

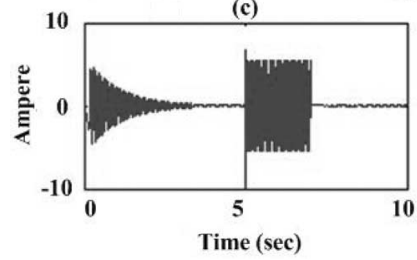

(d)
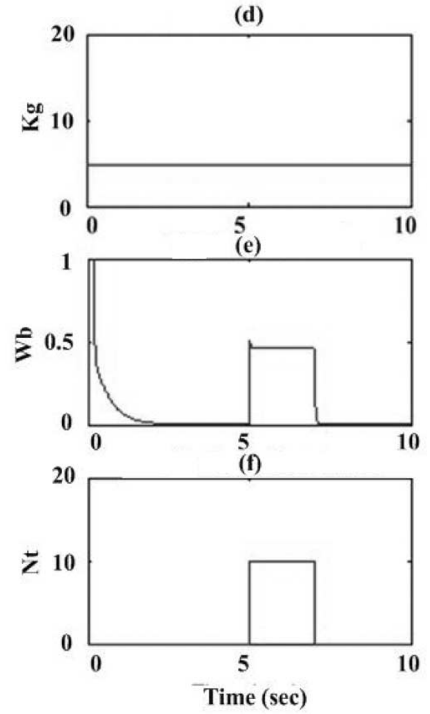

Fig. 4. $p_{d}=10\left(1-e^{-2 t}\right) \mathrm{cm}$ with $10-\mathrm{N}$ disturbance.

\section{B. Results of Adaptive Backstepping Controller Design}

In order to confirm the performance of the controller design in Section IV. We proposed several kinds of desired position trajectories, including exponential command with disturbance, and sinusoidal function, respectively. All the results are shown in Figs. 4 and 5. All these position tracking errors will approach to zero when time goes to infinity.

Remark: Compare the simulated results of the two kinds of approaches. The adaptive backstepping controller has better performance, e.g., lower position error, save power (effectively excited current and flux), etc., because the adaptive backstepping controller does not require acceleration signals.

\section{EXPERIMENTAL RESULTS}

In this section, extensive experiments are done with a 4-ploe, 3-phase linear induction motor with a $Y$-connected primary, manufactured by NORMAG Co. Detailed parameters and specification will be found in Table I and Fig. 6 shows a photograph of the constituted experimental equipment. The power stage of the motor driver uses a IGBT module, and the PWM drive signals are generated by a $10 \mathrm{KHz}$ SPWM with a $2.5 \mu$ s dead-time protection circuit. The software we adopt in Simulink and Matlab, an excellent product of The MathWorks Inc., Natick, MA. In addition, we use Simu-Drive to combine the motor control card with the Simulink/Real Time Workshop. 
(a)
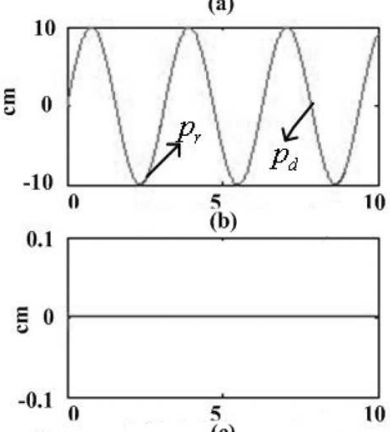

(c)

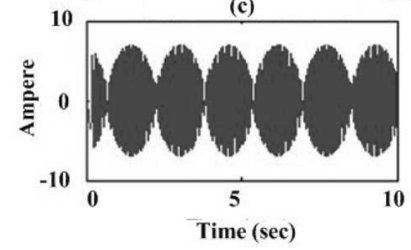

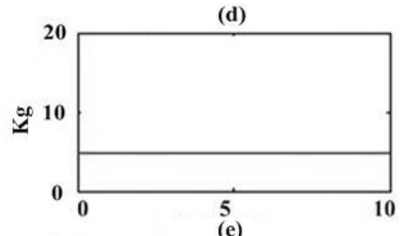
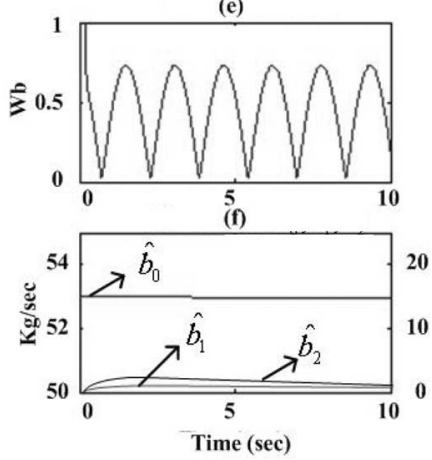

Fig. 5. $p_{d}=10 \sin (2 t) \mathrm{cm}$.

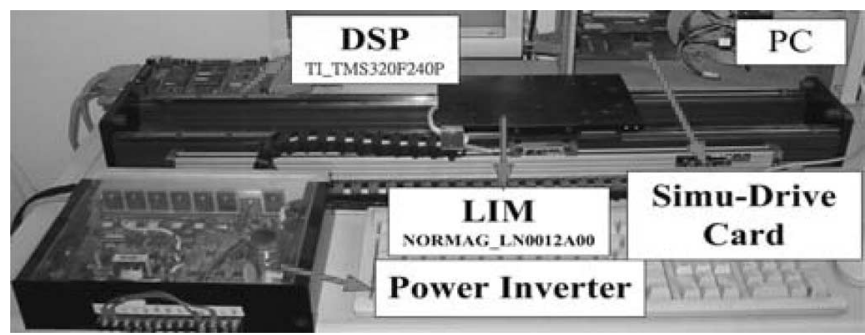

Fig. 6. Experimental equipment of LIM.

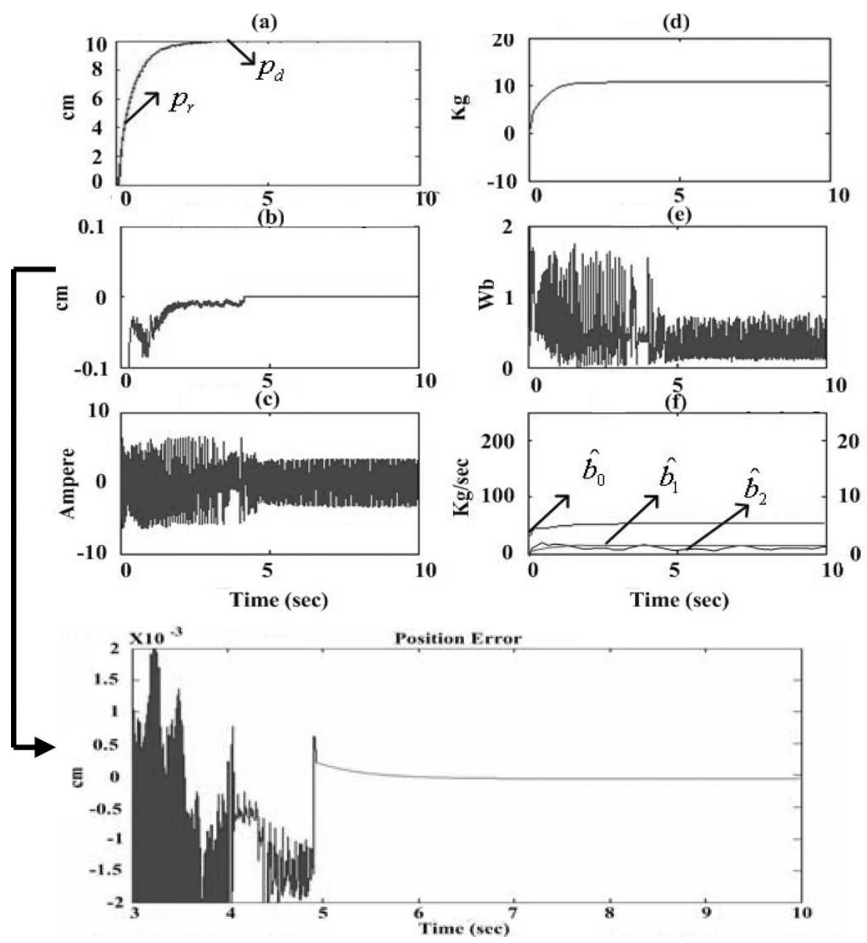

Fig. 7. $p_{d}=10\left(1-e^{-2 t}\right) \mathrm{cm} /$.

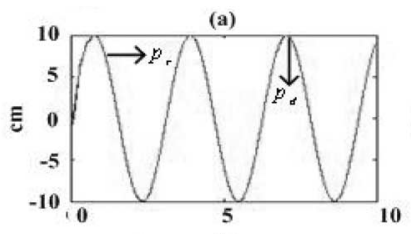

(b)
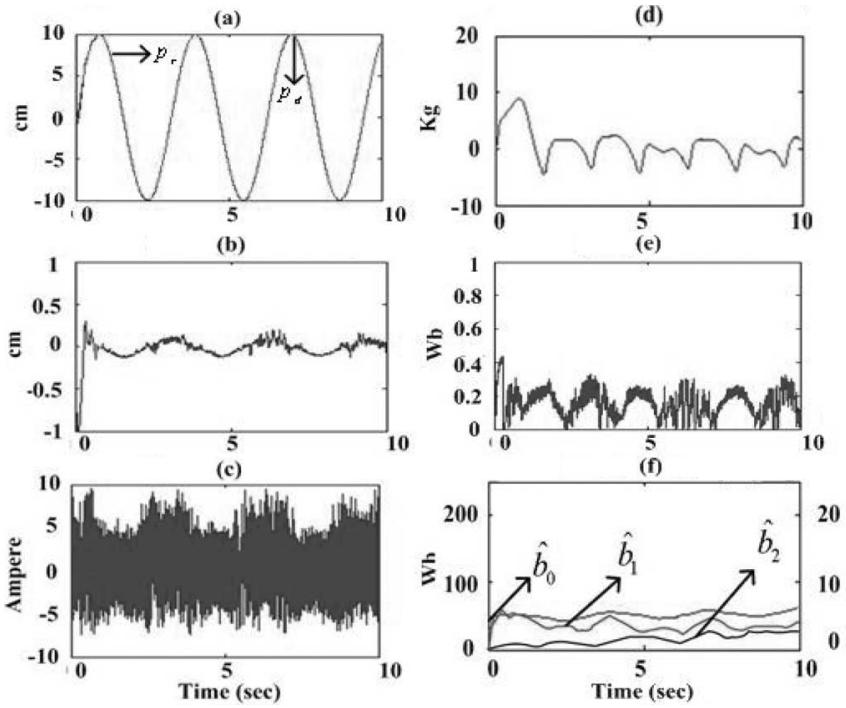

Fig. 8. $p_{d}=10 \sin (2 t) \mathrm{cm} / \mathrm{sec}$.
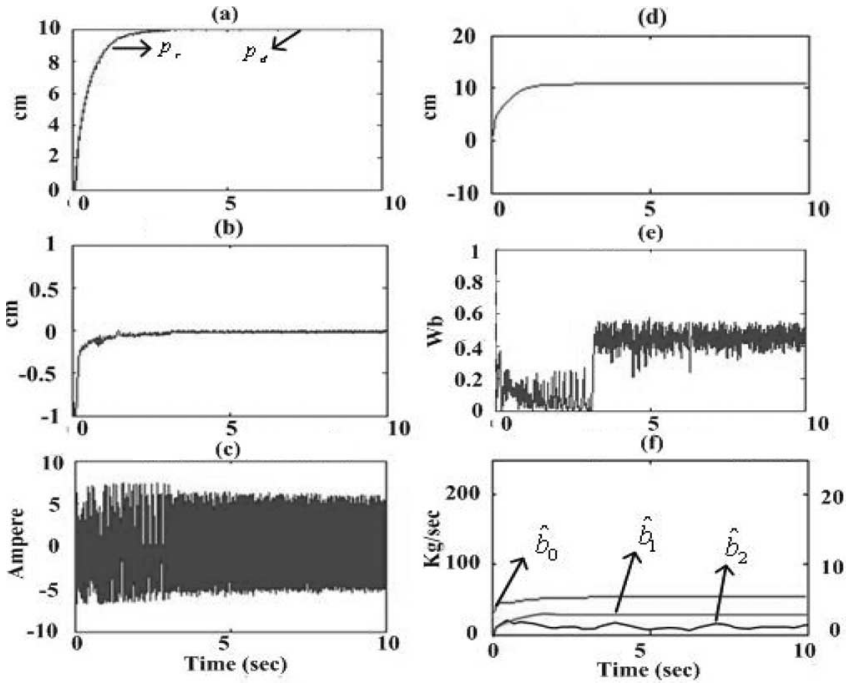

Fig. 9. $p_{d}=10\left(1-e^{-2 t}\right) \mathrm{cm}$.

\section{A. Results of Nonlinear Adaptive Controller Designfor Position Tracking}

In this section, we make a series of experiments on the adaptive controller design proposed in Section III. For the exponential type of desired trajectory in Fig. 7, the error less than $\pm 1 \mu \mathrm{m}$. In Fig. 8, we adopt sinusoidal trajectories, and the steady error is within $\pm 0.5 . \mathrm{mm}$

Remark: For experiment of the exponential type of desired trajectory in Fig. 7, we found chattering phenomenon in the steady state. In order to eliminate such phenomenon, we propose a PI control scheme, which, like a low-pass filter, can cut off high frequency behavior so that the position tracking error is kept within $\pm 100 \mu \mathrm{m}$. But eventually, such an arrangement will truly render the error less than $\pm 1 \mu \mathrm{m}$. 

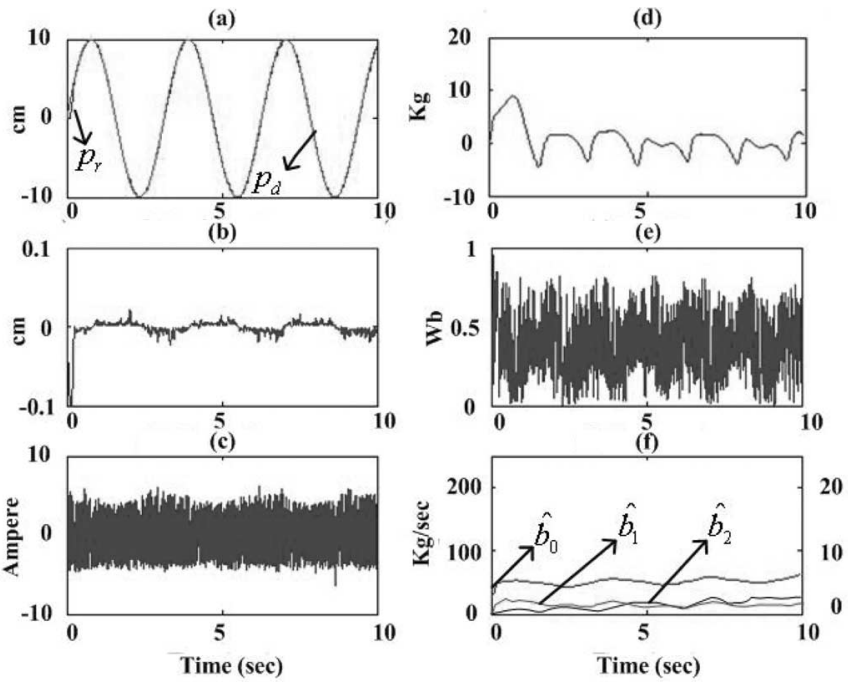

Fig. 10. $p_{d}=10 \sin (2 t) \mathrm{cm}$.

\section{B. Results of Adaptive Backstepping Controller for Position Tracking}

For the same position tracking problem, to validate the effectiveness of the adaptive backstepping controller, we provide several kinds of desired position trajectories as before. For the exponential trajectories in Fig. 9, we found chattering phenomenon in the steady-state error within $\pm 50 \mu \mathrm{m}$. In Fig. 10, we adopt sinusoidal trajectories, the steady error is within $\pm 0.7 \mathrm{~mm}$ and the similar kind of error convergence can still be guaranteed.

Remark: From above experimental results, we found they are very consistent with simulated results. The adaptive backstepping approach has lower position error and lower power loss than nonlinear adaptive approach.

\section{CONCLUSION}

In this paper, we have proposed two kinds of adaptive position tracking controller for the linear induction motor with sixth-order nonlinear dynamic model which is controlled by the primary voltage source. To cope with the uncertainty part of the linear induction motor, i.e., friction, end effect, payload, and inductance, we design our controller based on an appropriate nonlinear transformation. Due to inaccessibility to the flux in general, flux observers have been introduced. A stability analysis based on Lyapunov theory is performed to guarantee that the closed-loop system is stable. Finally, both the simulation and experimental results confirm the effectiveness of our control design.

\section{REFERENCES}

[1] S. Yamamura, Theory of Linear Induction Motors. New York: Wiley, 1972.

[2] I. Takahashi and Y. Ide, "Decoupling control of thrust and attractive force of a LIM using a space vector control inverter," IEEE Trans. Ind. Appl., vol. 29, no. 1, pp. 161-167, Jan./Feb. 1993.

[3] D. Dolinar, G. Stumberger, and B. Grcar, "Calculation of the linear induction motor model parameters using finite elements," IEEE Trans. Magn., vol. 34, no. 5, pp. 3640-3643, Sep. 1998.
[4] M. Mirsalim, A. Doroudi, and J. S. Moghani, "Obtaining the operating characteristics of linear induction motors: A new approach," IEEE Trans. Magn., vol. 38, no. 2, pp. 1365-1370, Mar. 2002.

[5] Z. Zhang, T. R. Eastham, and G. E. Dawson, "LIM dynamic performance assessment from parameter identification," in Conf. Rec. IEEE IAS Annu. Meeting, 1993, vol. 2, pp. 1047-1051.

[6] J. H. Sung and K. Nam, "A new approach to vector control for a linear induction motor considering end effects," in Conf. Rec. IEEE IAS Annu. Meeting, 1999, pp. 2284-2289.

[7] J. H. Lee, S. C. Ahn, and D. S. Hyun, "Dynamic characteristic analysis of vector controlled LIM by finite element method and experiment," in Conf. Rec. IEEE IAS Annu. Meeting, 1998, pp. 799-806.

[8] B. Kwon, K. Woo, and S. Kim, "Finite element analysis of direct thrustcontrolled linear induction motor," IEEE Trans. Magn., vol. 35, no. 3, pp. 1306-1309, May 1999.

[9] R.C. Creppe et al., "Dynamic behavior of a linear induction motor," in Proc. Mediterr. Electrotech. Conf., vol. 2, 1998, pp. 1047-1051.

[10] A. Shanmugasundaram and M. Rangasamy, "Control of compensation in linear induction motors," Proc. Inst. Electr. Eng., vol. 135, pp. 22-32, 1988.

[11] F. J. Lin and C. C. Lee, "Adaptive backstepping control for linear induction motor drive to track periodic reference," Proc. Inst. Electr. Eng.-Electr. Power Appl., vol. 147, no. 6, pp. 449-458, 2000.

[12] P. C. Krause, Analysis of Electric Machinery. New York: McGraw-Hill, 1986.

[13] H. T. Lee, L. C. Fu, and H. S. Huang, "Sensorless speed tracking control of induction motor with unknown torque based on maximum power transfer," IEEE Trans. Ind. Electron., vol. 49, no. 4, pp. 911-924, Aug. 2002.

[14] S. Sastry and M. Bodon, Adaptive Control: Stability, Convergence, and Robustness. Englewood Cliffs, NJ: Prentice-Hall, 1989.

[15] C. C. de Wit, H. Olsson, K. J. Astrom, and P. Lischinsky, "A new model for control of systems with friction," IEEE Trans. Autom. Control, vol. 40, no. 3, pp. 419-425, Mar. 1995.

[16] Y. Tan and I. Kanellakopoulos, "Adaptive nonlinear friction compensation with parametric uncertainties," in Proc. 1999 Amer. Control Conf., San Diego, CA, pp. 2511-2515.

[17] Y. Tan, J. Chang, and H. Tan, "Adaptive nonlinear friction compensation with parametric uncertainties," in Proc. 2000 Amer. Control Conf., Chicago, pp. 2511-2515.

[18] Y. Zhu and P. R. Pagilla, "Adaptive controller and observer design for a class of nonlinear system," Trans. ASME, J. Dyn. Syst., Meas. Control, vol. 128, pp. 712-717, 2006.

[19] H. Olsson and K. J. Åström, "Friction generated limit cycles," IEEE Trans. Control Syst. Technol., vol. 9, no. 4, pp. 629-636, Jul. 2001.

[20] Y. Zhu and P. R. Pagilla, "Static and dynamic friction compensation in trajectory tracking control of robots," in Proc. IEEE Int. Conf. Robot. Autom., 2002, vol. 3, pp. 2644-2649.

[21] N. Fujii and T. Harada, "Simple end effect compensator for linear induction motor," IEEE Trans. Magn., vol. 38, no. 5, pp. 3270-3272, Sep. 2002.

[22] I. E. Davidson and J. F. Gieras, "Performance analysis of a shaded-pole linear induction motor using symmetrical components, field analysis, and finite element method," IEEE Trans. Energy Convers., vol. 15, no. 1, pp. 24-29, Mar. 2000.

[23] F. J. Lin, R. J. Wai, W. D. Chou, and S. P. Hsu, "Adaptive backstepping control using recurrent neural network for linear induction motor drive," IEEE Trans. Ind. Electron., vol. 49, no. 1, pp. 134-146, Feb. 2002.

[24] R. J. Wai and W. K. Liu, "Nonlinear control for linear induction motor servo drive," IEEE Trans. Ind. Electron., vol. 50, no. 5, pp. 920-935, Oct. 2003.

[25] H. Amirkhani and A. Shoulaie, "Online control of thrust and flux in linear induction motors," Proc. Inst.Electr. Eng._Electr. Power Appl., vol. 150, no. 5, pp. 515-520, 2003.

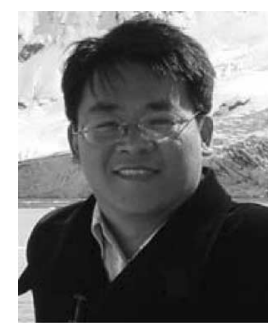

Chin-I Huang (S'01) was born in Hsinchu, Taiwan, R.O.C., in 1973. He received the M.S. degree in electrical engineering from Chung Yuan Christian University, Chung Li, Taiwan, in 2000 . He is currently working toward the Ph.D. degree in electrical engineering at National Taiwan University, Taipei, Taiwan.

His research interests include electrical drives, robotics, virtual reality, and nonlinear control theory and applications. 


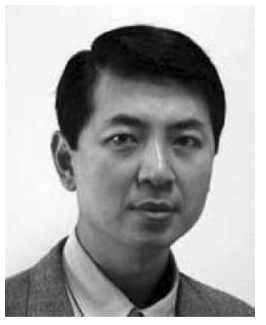

Li-Chen Fu (S'85-M'88-SM'02-F'04) received the B.S. degree from National Taiwan University, Taipei, Taiwan, R.O.C., in 1981, and the M.S. and Ph.D. degrees from the University of California, Berkeley, in 1985 and 1987, respectively.

Since 1987, he has been a member of the faculty, and is currently a Professor in the Department of Electrical Engineering and Department of Computer Science and Information Engineering, National Taiwan University, where he is also currently the Secretary General of the University. His current research interests include robotics, FMS scheduling, shop floor control, home automation, visual detection and tracking, e-commerce, and control theory and applications. He has been the Editor of the Journal of Control and Systems Technology and the Associate Editor of the prestigious control journal, Automatica. In 1999, he became the Editor-in-Chief of the Asian Journal of Control.

Prof. Fu is a Senior Member of the IEEE Robotics and Automation and IEEE Automatic Control Societies. From 2004 to 2005, he was an AdCom Member of the IEEE Robotics and Automation Society. He was the Program Chair of the IEEE International Conference on Robotics and Automation (ICRA) in 2003 and the Program Chair of the IEEE International Conference on Control Applications (CCA) in 2004. 